\begin{tabular}{c} 
International Journal of Scientific World, 5(2)(2017) $141-144$ \\
International Journal of Scientific World \\
SPC \\
Website: $\begin{array}{c}\text { www.sciencepubco.com/index.php/IJSW } \\
\text { doi: } 10.14419 / i j s w . v 5 i 2.8371 \\
\text { Research paper }\end{array}$ \\
\hline
\end{tabular}

\title{
On estimation of Frechet distribution with known shape using Bayesian analysis under informative priors
}

\author{
Wajiha Nasir ${ }^{*}$ \\ ${ }^{1}$ Govt. College Women University, Sialkot \\ ${ }^{*}$ Corresponding author E-mail: wajiha.nasir@gcwus.edu.pk
}

\begin{abstract}
In this study, Frechet distribution has been studied by using Bayesian analysis. Posterior distribution has been derived by using gamma and exponential. Bayes estimators and their posterior risks has been derived using five different loss functions. Elicitation of hyperparameters has been done by using prior predictive distributions. Simulation study is carried out to study the behavior of posterior distribution. Quasi quadratic loss function and exponential prior are found better among all.
\end{abstract}

Keywords: Bayes estimator, posterior risks, Loss function, extreme value, posterior, prior, elicitation

\section{Introduction}

Frechet distribution is from of the well-known family of distribution known as extreme value distribution. It is recognized as extreme value type-II distribution and also extreme value distribution of maxima. The three parameters Frechet distribution has been estimated by Gumbel (1965). Asymptomatic distribution of extreme stock market return follows Frechet distribution showed by Longin (1996). The parameters of Frechet distribution has been estimated by Mubarak (2012). Frechet distribution has been used to measure ultrasonic pulse velocity in concrete by Chatterjee and Chatterjee (2012). Frechet distribution has been used to study analysis of accelerated life testing by using geometric process by Shahab and Islam (2013).The parameters of Frechet distribution has been estimated by using maximum likelihood estimation and least squares under Type-II censored samples by Abbas and Tang (2013).Frechet distribution with known shape under different sampling techniques has been studied by Sindhu et al. (2014). Nasir and Aslam (2015) has studied the estimation of shape parameter with known scale via Bayesian analysis under informative priors. Nasir et al. (2015) have studied the estimation of shape parameter with known scale using Bayesian analysis by informative priors. The scale parameter of Frechet distribution has been estimated by Best Linear Unbiased Estimate (BLUE) and Nearly Best Linear Unbiased Estimate using lower record values by Tayyab et al. (2015). The parameters of Frechet distribution has been estimated by using maximum likelihood method and asymptotic confidence interval estimates by using progressive type-II censored data by Shahab et al. (2015).

A random variable $\mathrm{X}$ is said to have a Frechet distribution if its p.d.f. has following form

$$
\begin{aligned}
& f(\mathrm{x} ; \phi, \gamma)=\varphi \gamma^{\varphi}\left(\frac{1}{\mathrm{x}}\right)^{\varphi} e^{-\left(\frac{\gamma}{\mathrm{x}}\right)^{\varphi},}, 0<x<\infty, \\
& 0<\varphi<\infty, 0<\gamma<\infty
\end{aligned}
$$

Where where $\varphi$ is shape parameter and $\gamma$ is scale parameter. In this paper we see the behavior of scale parameter under Bayesian analysis by assuming $\varphi=1$. By considering $\varphi=1$ the p.d.f. for Frechet distribution reduces to following form

$f(\mathrm{x} ; 1, \gamma)=\gamma\left(\frac{1}{\mathrm{x}}\right) e^{-\left(\frac{\gamma}{\mathrm{x}}\right)}, 0<x<\infty, 0<\gamma<\infty$

\section{Bayesian Analysis Using complete samples}

In this section, Bayesian analysis under complete samples technique has been utilized. Posterior distribution has been derived under informative prior (Gamma, inverse levy and exponential). The Bayes estimators and their corresponding risks are derived usingSquare error loss function (SELF), Precautionary Loss Function (PLF), Simple Precautionary Loss Function (SPLF), Weighted Loss Function (WLF), LINEX Loss function and trigonometric loss function (TTLF) different loss functions.

Let the random sample $x_{1}, x_{2}, \ldots x_{n}$ is assumed to be taken from Frechet distribution with known shape parameter $\varphi$ and assuming $\varphi=1$ and with unknown parameter. Then likelihood function is

$L(\mathrm{x}, \gamma)=\gamma^{n} \prod_{i=1}^{n}\left(\frac{1}{\mathrm{x}_{i}}\right) e^{-\gamma \sum_{i=1}^{n}\left[\frac{1}{\mathrm{x}_{i}}\right]}$

The Gamma distribution is used as informative prior with hyperparameters $a$ and $b$ is

$p(\gamma)=\frac{\mathrm{b}^{\mathrm{a}}}{\Gamma(a)} \gamma^{a-1} e^{-b \gamma}, 0<\gamma<\infty, \mathrm{a}, \mathrm{b}>0$.

Now, the posterior distribution of $\gamma$ using 3 and 4 is

$p(\gamma \mid \underline{x}) \propto \gamma^{(a+n)-1} e^{-\gamma\left(b+\sum_{i=1}^{n}\left[\frac{1}{x_{i}}\right]\right)}$, 
So, $\gamma \mid \mathrm{x} \sim \operatorname{Gamma}\left(\alpha_{1}, \beta_{1}\right)$, where $\alpha_{1}=a+n$ and $\beta_{1}=b+\sum_{i=1}^{n} \frac{1}{x_{i}}$. The Bayes estimator and posterior risks under SELF, PLF, WLF, LLF, QQLF and TTLF using gamma prior are

$$
\begin{aligned}
& \hat{\gamma}_{S E L F}=\frac{a+n}{b+\sum_{i=1}^{n} \frac{1}{x_{i}}}, \rho\left(\hat{\gamma}_{S E L F}\right)=\frac{a+n}{\left(b+\sum_{i=1}^{n} \frac{1}{x_{i}}\right)^{2}}, \\
& \hat{\gamma}_{P L F}=\frac{\sqrt{(a+n+1)(a+n+2)}}{\left(b+\sum_{i=1}^{n} \frac{1}{x_{i}}\right)}, \\
& \rho\left(\hat{\gamma}_{P L F}\right)=2\left(\frac{\sqrt{(a+n+1)(a+n+2)}-(a+n)}{b+\sum_{i=1}^{n} \frac{1}{x_{i}}}\right) \text {, } \\
& \hat{\gamma}_{W L F}=\frac{a+n-1}{b+\sum_{i=1}^{n} \frac{1}{x_{i}}}, \rho\left(\hat{\gamma}_{W L F}\right)=\frac{1}{b+\sum_{i=1}^{n} \frac{1}{x_{i}}}, \\
& \hat{\gamma}_{L L F}=-\frac{1}{t} \log \left(1+\frac{t}{b+\sum_{i=1}^{n}\left(\frac{1}{x_{i}}\right)}\right)^{-(a+n)} \\
& \rho\left(\hat{\gamma}_{L L F}\right)=-\frac{1}{t} \log \left(1+\frac{t}{b+\sum_{i=1}^{n}\left(\frac{1}{x_{i}}\right)}\right)^{-(a+n)} \\
& +t\left(\frac{a+n}{b+\sum_{i=1}^{n}\left(\frac{1}{x_{i}}\right)}\right)
\end{aligned}
$$$$
\hat{\gamma}_{T T L F}=-\frac{1}{2} \log \left(\frac{\left(1-\frac{t}{b+\sum_{i=1}^{n}\left(\frac{1}{x_{i}}\right)}\right)^{-(a+n)}}{\left(1+\frac{t}{b+\sum_{i=1}^{n}\left(\frac{1}{x_{i}}\right)}\right)^{-(a+n)}}\right) \text {, }
$$$$
\rho\left(\hat{\gamma}_{T T L F}\right)=\sqrt{\left(1-\frac{t}{b+\sum_{i=1}^{n}\left(\frac{1}{x_{i}}\right)}\right)^{-(a+n)}}
$$$$
\times \sqrt{\left(1+\frac{t}{b+\sum_{i=1}^{n}\left(\frac{1}{x_{i}}\right)}\right)^{-(a+n)}}-1
$$

. The inverse levy prior is assumed as informative prior with hyperparameter $k$ is

$p(\gamma)=\sqrt{\frac{k}{2 \pi}} \gamma^{-\frac{1}{2}} e^{\frac{l}{2} \gamma} 0<\gamma<\infty, k>0$

The posterior distribution using 3 and 6 is

$p(\gamma \mid) \propto \gamma^{\left(n+\frac{1}{2}\right)-1} e^{-\gamma\left(\frac{k}{2}+\sum_{i=1}^{n}\left[\frac{1}{x_{i}}\right]\right)}$,
So, $\gamma \mid \mathrm{x} \sim \operatorname{Gamma}\left(\alpha_{2}, \beta_{2}\right)$, where $\alpha_{1}=n+\frac{1}{2}$ and $\beta_{1}=\frac{k}{2}+\sum_{i=1}^{n} \frac{1}{x_{i}}$.

The Bayes estimator and posterior risks under SELF, PLF, WLF, LLF, QQLF and TTLF using 7 are

$$
\begin{aligned}
& \hat{\gamma}_{S E L F}=\frac{2 n+1}{l+2 \sum_{i=1}^{n} \frac{1}{x_{i}}}, \rho\left(\hat{\gamma}_{S E L F}\right)=\frac{4 n+2}{\left(l+2 \sum_{i=1}^{n} \frac{1}{x_{i}}\right)^{2}}, \\
& \hat{\gamma}_{P L F}=\frac{\sqrt{(2 n+1)(2 n+3)}}{\left(l+2 \sum_{i=1}^{n} \frac{1}{x_{i}}\right)} \\
& \rho\left(\hat{\gamma}_{P L F}\right)=2\left(\frac{\sqrt{(2 n+1)(2 n+3)}-\left(n+\frac{1}{2}\right)}{l+2 \sum_{i=1}^{n} \frac{1}{x_{i}}}\right),
\end{aligned}
$$$$
\hat{\gamma}_{W L F}=\frac{2 n-3}{l+2 \sum_{i=1}^{n} \frac{1}{x_{i}}}, \rho\left(\hat{\gamma}_{W L F}\right)=\frac{2}{l+2 \sum_{i=1}^{n} \frac{1}{x_{i}}},
$$$$
\hat{\gamma}_{L L F}=-\frac{1}{t} \log \left(1+\frac{t}{\frac{l}{2}+\sum_{i=1}^{n}\left(\frac{1}{x_{i}}\right)}\right)^{-\left(n+\frac{1}{2}\right)},
$$$$
\begin{aligned}
\rho\left(\hat{\gamma}_{L L F}\right) & =-\frac{1}{t} \log \left(1+\frac{t}{\frac{l}{2}+\sum_{i=1}^{n}\left(\frac{1}{x_{i}}\right)}\right)^{-\left(n+\frac{1}{2}\right)} \\
& +t\left(\frac{n+\frac{1}{2}}{\frac{l}{2}+\sum_{i=1}^{n}\left(\frac{1}{x_{i}}\right)}\right)
\end{aligned}
$$$$
\hat{\gamma}_{T T L F}=-\frac{1}{2} \log \left(\frac{\left(1-\frac{t}{\frac{l}{2}+\sum_{i=1}^{n}\left(\frac{1}{x_{i}}\right)}\right)^{-\left(n+\frac{1}{2}\right)}}{\left(1+\frac{t}{\frac{l}{2}+\sum_{i=1}^{n}\left(\frac{1}{x_{i}}\right)}\right)^{-\left(n+\frac{1}{2}\right)}}\right) \text {, }
$$

$$
\begin{aligned}
\rho\left(\hat{\gamma}_{T T L F}\right) & =\sqrt{\left(1-\frac{t}{\frac{l}{2}+\sum_{i=1}^{n}\left(\frac{1}{x_{i}}\right)}\right)^{-\left(n+\frac{1}{2}\right)}} \\
& \times \sqrt{\left(1+\frac{t}{\frac{l}{2}+\sum_{i=1}^{n}\left(\frac{1}{x_{i}}\right)}\right)^{-\left(n+\frac{1}{2}\right)}}-1 .
\end{aligned}
$$

The exponential prior is assumed as informative prior with hyperparameter $f$ is

$p(\gamma)=f e^{-f \gamma}, 0<\gamma<\infty, f>0$.

The posterior distribution using 3 and 8 is

$p(\gamma \mid \underline{\mathrm{x}}) \propto \gamma^{(n+1)-1} e^{-\gamma\left(f+\sum_{i=1}^{n}\left[\frac{1}{\mathrm{x}_{i}}\right]\right)}$, 
So, $\gamma \mid \mathrm{x} \sim \operatorname{Gamma}\left(\alpha_{3}, \beta_{3}\right)$, where $\alpha_{1}=n+1 \operatorname{and} \beta_{3}=f+\sum_{i=1}^{n} \frac{1}{x_{i}}$.

The Bayes estimator and posterior risks under SELF, PLF, WLF, LLF, QQLF and TTLF using 9 are

$$
\begin{aligned}
& \hat{\gamma}_{S E L F}=\frac{n+1}{f+\sum_{i=1}^{n} \frac{1}{x_{i}}}, \rho\left(\hat{\gamma}_{S E L F}\right)=\frac{n+1}{\left(f+\sum_{i=1}^{n} \frac{1}{x_{i}}\right)^{2}}, \\
& \hat{\gamma}_{P L F}=\frac{\sqrt{(n+2)(n+3)}}{\left(f+\sum_{i=1}^{n} \frac{1}{x_{i}}\right)}, \\
& \rho\left(\hat{\gamma}_{P L F}\right)=2\left(\frac{\sqrt{(n+2)(n+3)}-(n+1)}{f+\sum_{i=1}^{n} \frac{1}{x_{i}}}\right), \\
& \hat{\gamma}_{W L F}=\frac{n}{f+\sum_{i=1}^{n} \frac{1}{x_{i}}}, \rho\left(\hat{\gamma}_{W L F}\right)=\frac{1}{f+\sum_{i=1}^{n} \frac{1}{x_{i}}}, \\
& \hat{\gamma}_{L L F}=-\frac{1}{t} \log \left(1+\frac{t}{f+\sum_{i=1}^{n}\left(\frac{1}{x_{i}}\right)}\right)^{-(n+1)}, \\
& \rho\left(\hat{\gamma}_{L L F}\right)=-\frac{1}{t} \log \left(1+\frac{t}{f+\sum_{i=1}^{n}\left(\frac{1}{x_{i}}\right)}\right)^{-(n+1)} \\
& +t\left(\frac{n+1}{f+\sum_{i=1}^{n}\left(\frac{1}{x_{i}}\right)}\right)
\end{aligned}
$$$$
\hat{\gamma}_{T T L F}=-\frac{1}{2} \log \left(\frac{\left(1-\frac{t}{f+\sum_{i=1}^{n}\left(\frac{1}{x_{i}}\right)}\right)^{-(1+n)}}{\left(1+\frac{t}{f+\sum_{i=1}^{n}\left(\frac{1}{x_{i}}\right)}\right)^{-(1+n)}}\right) \text {, }
$$$$
\begin{aligned}
\rho\left(\hat{\gamma}_{T T L F}\right) & =\sqrt{\left(1-\frac{t}{f+\sum_{i=1}^{n}\left(\frac{1}{x_{i}}\right)}\right)^{-(1+n)}} \\
& \times \sqrt{\left(1+\frac{t}{f+\sum_{i=1}^{n}\left(\frac{1}{x_{i}}\right)}\right)^{-(1+n)}}-1,
\end{aligned}
$$

\section{Elicitation of hyper-parameters}

In this section, elicitation of hyper-parameters has been carried out. Elicitation of hyper-parameters is a pivotal stride. It makes it simple for us to understand what the specialists have faith in and what their assessments are. We have used method proposed by Aslam (2002).
Table 1: BEs and PRs for $\gamma=2$ using Gamma prior

\begin{tabular}{c|ccccc}
\hline \multirow{2}{*}{$\mathrm{n}$} & \multicolumn{3}{|c}{ Gamma prior } & & \\
\cline { 2 - 5 } & 100 & 300 & 500 & 1000 \\
\hline \multirow{2}{*}{ SELF } & 2.1255 & 2.0821 & 2.0584 & 2.0355 \\
& $(0.0408)$ & $(0.0134)$ & $(0.0080)$ & $(0.0040)$ \\
\hline \multirow{2}{*}{ PLF } & 2.1594 & 2.1016 & 2.0563 & 2.0494 \\
& $(0.0198)$ & $(0.0066)$ & $(0.0039)$ & $(0.0019)$ \\
\hline \multirow{2}{*}{ TTLF } & 1.9902 & 1.9986 & 1.9993 & 2.0002 \\
& $(0.0205)$ & $(0.0067)$ & $(0.0040)$ & $(0.0020)$ \\
\hline \multirow{2}{*}{ WLF } & 2.0626 & 2.0381 & 2.0279 & 2.0197 \\
& $(0.0198)$ & $(0.0067)$ & $(0.0040)$ & $(0.0020)$ \\
\hline \multirow{2}{*}{ LLF } & 2.1229 & 2.0752 & 2.0563 & 2.0436 \\
& $(0.0201)$ & $(0.0066)$ & $(0.0040)$ & $(0.0031)$ \\
\hline
\end{tabular}

For this method, prior predictive distribution has been derived by using following expression

$p(y \mid \underline{x})=\int_{-\infty}^{\infty} p(\gamma \mid \underline{x}) f(y ; \gamma) d \gamma$

The prior predictive under gamma, Inverse levy and exponential prior is

$p_{g}(y \mid \mathrm{x})=\frac{a b^{a}}{y\left(b+\frac{1}{y}\right)^{a+1}}$,

The value for $a=2.735684$ and $b=0.864107$ using 11 by the method of elicitation.

$p_{I L}(y \mid \mathrm{x})=\sqrt{\frac{k}{2 \pi}} \frac{1}{2\left(\frac{k}{2}+\frac{1}{y}\right)^{\frac{3}{2}}}$,

The value for $k=4.987213$ using 12 by the method of elicitation.

$p_{e}(y \mid \underline{\mathbf{x}})=\frac{f}{\left(f+\frac{1}{y}\right)^{2}}$,

The value for $f=2.02528$ using 13 by the method of elicitation.

\section{Simulation Study}

A simulation study has been led to assess the conduct and execution of various estimators. The estimators are compared by their posterior risks, the estimator with minimum posterior risks is considered better among all. The simulation studied has been done for variant values of parameters and sample sizes. The shape parameter $\gamma \in\{2,5,8\}$ and the sample size $n \in\{100,300,500,1000\}$ are assumed . The simulation study is done by Mathematica.

The simulation study was directed by three different priors and under six loss functions. The following tables shows the results for Bayes estimates and posterior risks. The risks are listed in parentheses. 
Table 2: BEs and PRs for $\gamma=5$ using Gamma prior

\begin{tabular}{c|ccccc}
\hline \multirow{2}{*}{$\mathrm{n}$} & \multicolumn{3}{|c}{ Gamma prior } & & \\
\cline { 2 - 5 } & 100 & 300 & 500 & 1000 \\
\hline \multirow{2}{*}{ SELF } & 4.8967 & 4.9378 & 4.9575 & 4.9746 \\
& $(0.2424)$ & $(0.0824)$ & $(0.0497)$ & $(0.0040)$ \\
\hline \multirow{2}{*}{ PLF } & 2.1594 & 2.1016 & 2.0563 & 2.0494 \\
& $(0.0198)$ & $(0.0066)$ & $(0.0039)$ & $(0.0249)$ \\
\hline \multirow{2}{*}{ TTLF } & 4.5826 & 4.7530 & 4.8285 & 4.8694 \\
& $(0.1295)$ & $(0.0422)$ & $(0.0315)$ & $(0.0125)$ \\
\hline \multirow{2}{*}{ WLF } & 4.7275 & 4.8316 & 4.8844 & 4.9228 \\
& $(0.0483)$ & $(0.0164)$ & $(0.0099)$ & $(0.0050)$ \\
\hline \multirow{2}{*}{ LLF } & 4.8984 & 4.9352 & 4.9620 & 4.9749 \\
& $(0.1173)$ & $(0.0408)$ & $(0.0308)$ & $(0.0124)$ \\
\hline
\end{tabular}

Table 3: BEs and PRs for $\gamma=2$ using Inverse levy prior

\begin{tabular}{cccccc}
\hline \multirow{2}{*}{$\mathrm{n}$} & \multicolumn{4}{|c}{ Inverse levy prior } \\
\cline { 2 - 5 } & 100 & 300 & 500 & 1000 \\
\hline \multirow{2}{*}{ SELF } & 1.9728 & 1.9924 & 1.9931 & 1.9978 \\
& $(0.0391)$ & $(0.0133)$ & $(0.0080)$ & $(0.0040)$ \\
\hline \multirow{2}{*}{ PLF } & 1.9857 & 1.9957 & 1.9967 & 2.9998 \\
& $(0.0196)$ & $(0.0066)$ & $(0.0040)$ & $(0.0021)$ \\
\hline \multirow{2}{*}{ TTLF } & 1.938 & 1.9771 & 1.9872 & 1.9932 \\
& $(0.0198)$ & $(0.0067)$ & $(0.0040)$ & $(0.0020)$ \\
\hline \multirow{2}{*}{ WLF } & 1.9564 & 1.9857 & 1.9915 & 1.9954 \\
& $(0.0197)$ & $(0.0066)$ & $(0.0040)$ & $(0.0020)$ \\
\hline \multirow{2}{*}{ LLF } & 1.9936 & 1.9778 & 1.9992 & 1.9998 \\
& $(0.0193)$ & $(0.0066)$ & $(0.0040)$ & $(0.0021)$ \\
\hline \multirow{5}{*}{}
\end{tabular}

Table 4: BEs and PRs for $\gamma=5$ using Inverse levy prior

\begin{tabular}{|c|c|c|c|c|}
\hline \multirow[b]{2}{*}{$\mathrm{n}$} & \multicolumn{4}{|c|}{ Inverse levy prior } \\
\hline & 100 & 300 & 500 & 1000 \\
\hline \multirow{2}{*}{ SELF } & 4.8967 & 4.9378 & 4.9575 & 4.9746 \\
\hline & $(0.2424)$ & $(0.0824)$ & $(0.0497)$ & $(0.0040)$ \\
\hline \multirow{2}{*}{ PLF } & 4.7657 & 4.9151 & 4.9501 & 4.9761 \\
\hline & $(0.0471)$ & $(0.0163)$ & $(0.0099)$ & $(0.0050)$ \\
\hline \multirow{2}{*}{ TTLF } & 4.5826 & 4.7530 & 4.8285 & 4.8694 \\
\hline & $(0.1295)$ & $(0.0422)$ & $(0.0315)$ & $(0.0125)$ \\
\hline \multirow{2}{*}{ WLF } & 4.7275 & 4.8316 & 4.8844 & 4.9228 \\
\hline & $(0.0483)$ & $(0.0164)$ & $(0.0099)$ & $(0.0050)$ \\
\hline \multirow{2}{*}{ LLF } & 4.8984 & 4.9352 & 4.9620 & 4.9749 \\
\hline & $(0.1173)$ & $(0.0408)$ & $(0.0308)$ & $(0.0124)$ \\
\hline
\end{tabular}

Table 5: BEs and PRs for $\gamma=5$ using Exponential prior

\begin{tabular}{|c|c|c|c|c|}
\hline \multirow[b]{2}{*}{$\mathrm{n}$} & \multicolumn{4}{|c|}{ Exponential prior } \\
\hline & 100 & 300 & 500 & 1000 \\
\hline \multirow{2}{*}{ SELF } & 4.8133 & 4.8133 & 4.8875 & 4.9423 \\
\hline & $(0.2000)$ & $(0.0772)$ & $(0.0478)$ & $(0.0244)$ \\
\hline \multirow{2}{*}{ PLF } & 4.4982 & 4.8201 & 4.8924 & 4.9464 \\
\hline & $(0.0442)$ & $(0.0160)$ & $(0.0097)$ & $(0.0049)$ \\
\hline \multirow{2}{*}{ TTLF } & 4.3921 & 4.7808 & 4.8686 & 4.9345 \\
\hline & $(0.1124)$ & $(0.0403)$ & $(0.0244)$ & $(0.0124)$ \\
\hline \multirow{2}{*}{ WLF } & 4.4355 & 4.4987 & 4.7987 & 4.9408 \\
\hline & $(0.0444)$ & $(0.0160)$ & $(0.0098)$ & $(0.0049)$ \\
\hline \multirow{2}{*}{ LLF } & 4.8308 & 4.8375 & 4.8975 & 4.9476 \\
\hline & $(0.1031)$ & $(0.0390)$ & $(0.0240)$ & $(0.0123)$ \\
\hline
\end{tabular}

Table 6: BEs and PRs for $\gamma=2$ using Exponential prior

\begin{tabular}{ccccc}
\hline \multirow{2}{*}{$\mathrm{n}$} & \multicolumn{4}{|c}{ Exponential prior } \\
\cline { 2 - 5 } & 100 & 300 & 500 & 1000 \\
\hline \multirow{2}{*}{ SELF } & 1.9325 & 1.9753 & 1.9881 & 1.9930 \\
& $(0.0373)$ & $(0.0130)$ & $(0.0079)$ & $(0.0040)$ \\
\hline \multirow{2}{*}{ PLF } & 1.9414 & 1.9802 & 1.9882 & 1.9941 \\
& $(0.0191)$ & $(0.0065)$ & $(0.0040)$ & $(0.0020)$ \\
\hline \multirow{2}{*}{ TTLF } & 1.8965 & 1.9712 & 1.9896 & 1.9958 \\
& $(0.0193)$ & $(0.0066)$ & $(0.0040)$ & $(0.0020)$ \\
\hline \multirow{2}{*}{ WLF } & 1.9146 & 1.9712 & 1.9823 & 1.9904 \\
& $(0.0191)$ & $(0.0066)$ & $(0.0040)$ & $(0.0020)$ \\
\hline \multirow{2}{*}{ LLF } & 1.9511 & 1.9855 & 1.9896 & 1.9904 \\
& $(0.0189)$ & $(0.0065)$ & $(0.0039)$ & $(0.0020)$ \\
\hline
\end{tabular}

\section{Conclusion}

Table 1-4 summarized the simulation studies. The result, we concluded from this study, by increasing sample size Bayes posterior risk decreases and Bayes estimator approaches to its true value of the parameter. By increasing the value of parameter Bayes posterior risk also increases. PLF performs best among all loss functions as its posterior risk is minimum as compared to all studied loss functions. Exponential performs better among all as its posterior risk is minimum as compare to all other loss functions.

\section{References}

[1] Gumbel, E. J. "A quick estimation of the parameters in Fréchet's distribution." Revue de l'Institut International de Statistique (1965) 349-363.

[2] Longin, Francois M. "The asymptotic distribution of extreme stock market returns." Journal of business (1996): 383-408.

[3] Mubarak, Mohamed. "Parameter estimation based on the frechet progressive type ii censored data with binomial removals." International Journal of Quality, Statistics, and Reliability 2012 (2011).

[4] Chatterjee, Abhijit, and Anindya Chatterjee. "Use of the Fréchet distribution for UPV measurements in concrete." NDT E International 52 (2012): 122-128.

[5] Shahab, Sana, and Ariful Islam. "Analysis of accelerated life testing using geometric process for frechet distribution." ANALYSIS 2.9 (2013).

[6] Abbas, Kamran, and Yincai Tang. "Estimation of Parameters for Frechet Distribution Based on Type-II Censored Samples." Caspian Journal of Applied Sciences Research 2.7 (2013).

[7] Sindhu, Tabassum Naz, Muhammad Aslam, and Navid Feroze. "On estimation of the Frechet Distribution with known shape under different sample schemes." International Journal of Intelligent Technologies and Applied Statistics 7.4 (2014): 353-368.

[8] Nasir, Wajiha, and Muhammad Aslam. "Bayes approach to study shape parameter of Frechet distribution." International Journal of Basic and Applied Sciences 4.3 (2015): 246-254.

[9] Nasir, Wajiha, Maria Qudsia and Muhammad Aslam. "Bayesian Analysis of Shape Parameter of Frechet distribution using Non- Informative Prior." Elixir Statistics 88 (2015): 36362-36366.

[10] Aslam, Muhammad. "An application of prior predictive distribution to elicit the prior density." Journal of Statistical Theory and applications 2.1 (2003): 70-83. 